\title{
An Investigation on Mobile Communication Technologies and Their Effects on Elderly
}

\author{
F. Sahar and S. T. Raza
}

\begin{abstract}
In Europe people lives in an increasingly ageing society and there are different technological solutions that help them in their self management. Elderly population has been growing from 264 million in 2009 to 416 million in 2050 (UN Population Division/DESA). We believe that developing easy to use mobile functions can be of great use to the elderly as they grow older. The focus of this study is to investigate what functionalities the diabetes type 2 patients require in future mobile phones. The study is based on interviews with health care professionals and survey with elderly type 2 . Interviews carried out with those health care professional who are dealing with type 2 patients and understand the problems which type 2 patients facing in the use mobile phones. The second part is survey, we distributed questionnaire to type 2 elderly although they use mobile phone or not but majorly we focused those elderly who are already users of mobile phones. The results of questionnaire will find out the satisfaction of elder persons with their current mobile phones and also reasons of from those who are not users of mobile phone. Questionnaire's results will better explain the functionalities thatneeds to rebuild.
\end{abstract}

Index Terms-Ageing, diabetes type 2, elderly, mobile communication technologies.

\section{INTRODUCTION}

The adoption of mobile phone is growing very fast, the main reasons of growing are autonomous communication, connect from anywhere anytime, and emergencies services. Stay in touch all the time, safety, and some job related issues were the major factors of early adoption of mobile phone in young adults. However, safety and security were the main issues for elderly in adoption of mobile phone [1]. The importance of mobile phone is increasing on daily basis because people need the safety, self actualization, support, enjoyment, and simplicity in operating mobile phones [1]. The population of elderly is growing continuously with a high ratio. In last 50 years the population of elder people is increased three times and it is expected that it will increased more than three times in next 50 years [2]. According to the world population agency [2] the population of elder's people were 205 million in 1950 which became 606 million in 2000 . The expected population of the world in 2050 will be 2 billion. The growth ratio of elder people is (1.9 percent) higher than the other age groups (1.2 percent). The difference between elderly and other ages is becoming larger with passage of time. In 1950 from every 12 persons 1 was of age 60 and 1 out of 20 was of age 65 or elder. But this ratio is increased in 2000 when from every 10 persons 1 was age 60 and from every 14 persons 1 was of age 65 or elder. Due to

Manuscript received January 9, 2013; revised April 19, 2013.

The authors were with Blekinge Institute of Technology, SE- 37179 Karlskrona, Sweden (e-mail: farrukhsahar@yahoo.com, tosif@live.com). the fast growing ratio it is expected that in year 2050, 1 person from every 5 will be the 60 and from every 6 person 1 will be 65 or above [2]. Diabetes type 2 is most common in all over the world, approximately $95 \%$ of diabetic are suffering from type 2. It starts after the age of 40 and elders. Overweighed people also effected from it, due to high cholesterol in body and high blood pressure. Diabetes type 2 has 3 stages, first is against insulin and then dying of cells lastly sugar level become high in blood. This type is non-insulin dependent, and people suffering from it, do not require injection of insulin frequently [3]. It can be controlled by balanced diet, good exercise, and oral medication. If the patients of this disease are not cured and diagnosed, it may cause serious complication or may be end to death. Awareness is key factor to avoid serious type of problems and by getting awareness, patient needs to care about diet. The symptoms of diabetes type 2 includes frequent urination, weight loss, slow healing of wounds, increased in hunger and thirst, and blurred vision [3]. Elderly requires a special care all the time, due to age sometime it becomes difficult for them to solve their basic problems. The self-management and control of diabetes in elderly sometimes goes out of control. It is difficult to put nursing staff with each elder person, to remove this problem it is required to motivate the elderly to use mobile phones, so they will get early rescue in case of emergency. The main focus of our study is on functionalities of mobile phone which elderly type 2 want in future mobile phones. Majority of the elderly are unaware from using mobile phones; we also figure out the reasons of unfamiliarity with mobile phones and motivate them to use mobile phones. The type 2 elderly who are using mobile phones and feeling any difficulty in operating will better guide us about future functionalities on the basis of their experience.

\section{QUESTIONNAIRE AND INTERVIEW}

This chapter is divided in two parts first explain the questionnaire and second describes the interviews. Questionnaire is an easy way of obtaining the information and public opinion about any specific topic. The perfection and quality is an essential part of questionnaire in designing phase. Second part is the analysis of two interviews which we conducted with healthcare professionals for the better understanding of problems of diabetes type 2 elderly.

\section{A. Questionnaire Planning}

Questionnaire is an inexpensive way together rich data [4]. The main purpose of questionnaire planning was to obtain quantitative data to analyze the current state of mobile phone usage in diabetes type 2 elderly. The major portion of our 
questionnaire depends on close ended questions. In questionnaire our targeted goal was to achieve the mobile phone functionality which elder people want in future mobile phones. We have also focused on problems which elderly facing in using mobile phones. We have planned to visit old homes and hospitals in Ronneby and Karlskrona to target diabetes type 2 elderly. After the successful completion of questionnaire, we will analyze them statistically and will get final results.

\section{B. Questionaire Distribution}

We visited the old home and health center in Ronneby and Karlskrona for distributing the questionnaire. The English questionnaire converted in Swedish language, so the targeted persons can easily understand the questionnaire and answer them accurately. The questionnaire was distributed to 12 persons who were the mobile phone users and 4 persons were chosen who do not use the mobile phone.

\section{Questionnaire Analysis}

After receiving the answers of questionnaires we performed quantitative analysis. Elder person's response against close ended questions is calculated.

1) Close Ended Questions: The first four parts contain close ended questions. The first part is acceptability of mobile phone, in first question, 50\% elder people responds that they prefer single button for both functionalities of connect and disconnect whereas $50 \%$ people says that they do not prefer single button. In question 2 of questionnaire, around $42 \%$ respondents said that they want all three incoming, outgoing, and missed call functionality in call log. But approximately 58\% elder people said they don't want all three functionality in call $\log$.

Third question was very important, which asks from the elder people's that what thing is most important for them when they purchase mobile phone. According to the responses functionality of the mobile phone is most important factor at the time of purchasing of mobile, $67 \%$ elder persons said that they consider functionality of mobile phone at the time of purchasing, 50\% respondents consider the outlook of mobile handset. The fashion and brand do not effect in purchasing, around 58\% respondents said they don't care fashion and $50 \%$ said they don't care brand while purchasing new mobile phone.

In question 4 of questionnaire, $50 \%$ elder persons replied that they like flip mobile phone and 50\% did not replied on it. For straight mobile phone $42 \%$ people responds in strongly agree and 50\% said it is not applicable in choice. $83 \%$ elder persons didn't showed interest in Slide mobile handset whereas only $17 \%$ strongly agreed that they like slide mobile phone.

In question what size of mobile phone they like, $50 \%$ of respondents said they like medium size of mobile phone. While around $41 \%$ of people like small size mobile phones and only approximately $9 \%$ elder people like large size mobile handsets. This ratio shows that mostly elder people likes medium size mobile phones. In sixth question, $67 \%$ elder persons were strongly agree that "Call" should be written on connect button, only $25 \%$ people said that it is not applicable. Only $8.33 \%$ replied that "Send" should be written on connect button and $75 \%$ respond with not applicable. This percentage is showing that majority of elder people like that
Call is more accurate word than Send on call connect button.

The majority of elder persons likes that "end" should be written on call disconnect button. Around 58\% responds in favor of it and only $25 \%$ strongly agrees that "stop" should be written on call disconnect button. In question 8 of questionnaire, mostly people said they prefer that Icons are the best way to show the main menu items but the difference between two decisions is not very high. $58 \%$ respondents gave response in favor of "Icons" whereas 50\% said they prefer "Names". Question 9 we asked from elders that which color of mobile phone they like, $83 \%$ elder persons responds that they prefer "Bright" colors of mobile phones whereas only $17 \%$ replied that they like "Dark". Statistics of this question showed that bright colors of mobile phones are much popular in elder people.

In question 10 of the questionnaire, we asked what size of mobile phone the like. The results of this question were almost same with little difference. $42 \%$ respondents said they like "small" size mobile phone, $33 \%$ prefers "Medium", and $25 \%$ elder persons favors large size mobile handsets. In second part of questionnaire "functionality of mobile phone elder people prefer" is asked.

Question 11 was very important question for elderly point of views; due to ageing factor elder people lose their control on body movement especially on their motor functions. So a central locking button on mobile phone attracts them a lot. $83 \%$ elder people respond that they prefer central locking button in future mobile phone whereas only $17 \%$ reject this functionality in future mobile phones. Question 12 in the questionnaire is also very important question with the point of view of future functionality of mobile phone. Question was asking about the emergency button for some critical conditions when elderly do not know the exact number from where he takes help. Emergency button facilitate them to contact immediately with health centre or care centre. $75 \%$ elder persons replied in favor of emergency button in future mobile phones and only $25 \%$ respondents answered against this button. Visual effects are one of the chronicle factor which increases with the age of persons. In diabetes type 2 elderly decrease of eyesight is common factor. In this question $50 \%$ elderly replied that they prefer large screen sizes of future mobile phones. $25 \%$ said they wand small screen size and only $17 \%$ responds about the medium size of screen.

The question 14 of the questionnaire is also very important question for type 2 elder persons, this question also helps in maintaining the font size of mobile phone according to the requirement of elder persons. $67 \%$ elder persons replied that they want large font sizes in future mobile phones whereas only $25 \%$ respondents said they prefer medium size fonts. Third part of close ended questionnaire contain 2 questions only, 15 th question was very simple. $67 \%$ elderly replied that they always carry mobile phone with then and $33 \%$ told that they do not carry mobile phone all the time with them.

In question 16 of the questionnaire, we asked that how much they use mobile phone other regular calls. Only 33\% elderly replied that they use mobile phone for SMS, $17 \%$ said they use it in some emergency situations. According the statistics of this question SMS is popular functionality in elder persons after the regular calls. The fourth part of close ended questionnaire asks the questions regarding the eyesight 
and some diabetic issues relating with mobile phone. In question 17 we asked do you face difficulties on focusing the small and large objects of mobile phone. $67 \%$ elder people responds with "Yes" where as 33\% said they do not feel and problem in focusing the objects.

Question 18 was very simple question, in this question we asked whether elderly facing any problem in distinguishing the colors or not. $100 \%$ respondents replied positive and there was not a single person who was facing color blindness. Question 19 was about the maintenance of diabetes using mobile phone or at what level mobile phone helps them in managing their diabetes. Only one person responds that his mobile phone helps him in managing diabetes. The rest of respondents said they did not use their mobile phone yet for managing diabetes.

2) Open Ended Questions: Open ended questions are the best method to get the opinions and views of public. An open ended question provides the freedom to express own ideas without any bound. Following are the answers of three open ended questions.

3) In question 20 out of 12 targeted persons 9 respondents did not answer the question. One respondent said, mobile phone must have some functionality regarding the diabetes. Mobile phone hold record of blood pressure, provide estimation of sugar level, and estimation of serum control. Another respondent suggested mobile should have features which help in managing diabetes. Mobile phone also contains the functionality which informs the patient about the medicine dosage time. The last respondent said mobile phone should be a multipurpose device. It should perform the functionality like diabetes checking.

Question 21 of questionnaire was about the problems user facing with current mobile phones. Five respondents did not replied about this question. 33\% respondents said they are facing short battery life. One respondent said buttons of mobile phone are hard in pushing. Another was facing the accidental dialing problems. In question 22 of the questionnaire around $67 \%$ respondents did not give any suggestion. Two elder persons said that battery life of mobile phone should be increased and charging should take less time in completion. One person replied that the response time of mobile phone should be decreased. Another elder person said that mobile phone must contain the initial knowledge about the diabetes and also contain facilities to control the diabetes.

\section{Elderly Who Do Not Use Mobile Phone}

We distributed the questionnaire to 4 elderly who do not use mobile phones. Questionnaire contains two open ended questions, first was about the reason and second was about functionality.

In first question, two elder people said that they do not use mobile phone because they don't like mobile phones. One respondent said, he don't use mobile phone due to ageing factors. He is much older and his eyesight becomes weaker so, he is not interested in using mobile phone. Fourth respondent said, I use mobile phone only in homes but I am not a regular user of mobile phone. All four respondents have ignored the question 2. Because they are not the user of mobile phone so how they knew the current functionalities and also how they can express about the future functionalities.

\section{E. Interviews}

Interview is one of the techniques used for collecting qualitative data [5].We selected two interviewees, to complete the interview part of the thesis work. The first interview was conducted with the Lisbeth who working as a Nurse in Ronneby health care centre. She is working from last 4 years with the diabetes patients. The second interview conducted with Manzoor Ahmed who is a senior medical officer in Pakistan and working with diabetes patients from last 3 years.

\section{F. Importance of Interviews}

We planned to conduct interviews with health care professionals to identify the problems which diabetes type 2 patients facing in communication. The main reason behind the interviews was to validate our study, and the results of questionnaires. The opinions of professionals are helpful in better understanding of problems of diabetes type 2 patients and their solutions.

\section{G. Interviews Planning and Design}

For conduction of interviews we have planned different times for interviews. We took the appointment from the healthcare professionals and reached on decided time. The time duration of each interview was 30 to 45 minutes. We tried to insert maximum questions in our interview to identify the problems of diabetes type 2 patients. We designed two types of questions for interviews; first one was preplanned or formal question and second was runtime questions or informal questions. Informal questions asked from professionals during the interview. We focused mainly those problems of diabetes type 2 elderly which directly relates with using of mobile phone or functionality of the mobile phone.

\section{H. Interviews 1}

First interview was conducted with a Lisbeth (nurse) in County of Blekinge, Sweden. She has been working with diabetes patient for 4 years. She identified there are about 650 diabetes patient are registered in hospital and two nurses are dealing with them but she has no idea how many diabetes type 2 patients are registered in county of Blekinge. She explained the effect of type 2 diabetes on patients, patients feel tired and some time become arrogant because of their sugar levels.

1) Communication Gap: According to her patients only meet with doctors in special cases and after the check up nurses take care of that patient according to doctors instructions. She accepted that there is a communication gap between patient and health care staff because most often patients try to hide the facts about diet and exercise but health care staff gets all facts after the test results. She said the common reason for visibility problem is age not the type 2 diabetes effects.

2) Visibility Problems: According to the Nurse diabetes patients do not take effective measures in starting phase when they feel that their eyesight is weakening. Their eyesight does not weak suddenly but it takes time and decrease slightly and takes 5 to 10 years. Doctor describes the visibility issues as vascular problems of eyes. According to him the patients who face vision problems some time cannot see properly because of the condition of lens. To overcome visibility problems 
screen sizes of the mobile phone should b increases so the diabetes type 2 elderly can view properly

She identified some physical impairment because of type 2 diabetes, they suffer kidney problem, get foot sours and also suffer from gangiria. She said diabetes type 2 patients do not face any hearing problems. According to her if diabetes patients have routine checkups of their eyes then they do not have any chances of color recognition problems in the future. She explained that diabetes patients do not face problems in touch sensitivity of hand fingers but they have problems in foots and to some extent their feet become senseless.

3) Mobile Phone Support in Diabetes: According to her mobile phones cannot help them in managing diabetes. In her views it will be a difficult task for them to check their blood sugar level using mobile phones. She said that most of the diabetes patients who spent most of their time in home do not have any mobile phone. The patients who are living at home have a special alarm for emergency cases so in her views the patients who are living at home do not prefer to use mobile phones to manage their diabetes. She is agree with emergency button in mobile phones, she said alarms are better in homes which connects with health care centers for emergencies but if this facility can be included in mobile phones then patients feel more relax in contacting the health care centers in any situation.

4) Mobile Phone and Text Size: She said mobile phone size matters but it mostly depends on how often patients use mobile phones and according to her the patients who are working mostly have mobile phones. She suggested that emergency button in mobile phones will be supportive if it will be simple to use. She argued that emergency button could be helpful in some situations because in emergency situations it will very difficult to find the mobile phone.

According to her mostly patients who visited hospital don not have mobile phones so they never received any response related to problems in using mobile phones. She prefers to have larger text size in mobile phones not only for type 2 diabetes patients but also for elderly because most often they face visibility problems. She said hospital contact with patients through letters but in case if they need them immediately then they have patients numbers in some case both mobile phone and land line numbers.

\section{Interview 2}

Second interview was conducted with a senior medical officer, MBBS doctor in Pakistan. Who was dealing with diabetes patients for last 3 years and working in hospital for more than 29 years. He said daily 250 diabetes patients get registered. He explained the effect of type 2 diabetes on patients; patients feel hyper glycemia, suffer heart disease, also disease related to kidneys like renal failure or nephropathy as well as some time pus albumin in urine.

He identified communication gap between patients and health care staff because of no proper awareness of disease and lack of education. Some time they come with complains and they take the test but most often they come at complicated stages not in start. Most of the patients use homeopathic medicines and try to avoid the actual treatment.

1) Visibility Issues: He said type 2 diabetes patients face visibility problems like refractive errors suffer from retinopathy; it is a vascular problem of eyes. He identified patient face vision problems such as most of them suffer from catric, it is the condition lens cannot see properly and some time chances of vision loss and in that cases they need to operate. He identified some physical impairment because of type 2 diabetes, such as they do not feel healthy, physically effect on foot, loss of sensation and pain. Moreover He said diabetes type 2 patients do not face any hearing problems. He explained diabetes also effect on color recognition sense of patients they suffer from color recognition problems, blurring of vision also retinopathy creates problem in critical cases they need to get operate. He explained sensitivity problems in diabetes type 2 patients some effect on hands, like feeling pins pinching in hands; they don't differentiate between hot and cold. Their foot become senseless and they do not feel and pain.

However according to the doctor mobile phone can be useful in managing diabetes such as making contact with health care staff, person having the mobile phone can get help easily by contacting with them and communication gap will be decrease. He suggested that more the simple mobile patients will like to have it, light weight, easy to use, simple buttons and features, large screen, these all will be helpful and attract to patients.

2) Emergency Button of Mobile Phone: According to him especially in large population's area emergency button will be effective to get help quickly without delay. He suggested that there should be additional function, that patient just get his sugar level in his mobile just by pressing the button, so he can provide the correct information on time to the doctor. According to him larger text size will be very effective for diabetes type 2 patients. He thinks that Large text size is easy to read they can feel problem with small text because of catric, so they may be avoid to use the mobile so he think it is better having large text on mobile.

3) Mobile Phone Use: He analyzed that most of the old age do not have mobile phones; they even do not have idea how to use them. He said that from last 10 years trend are changed people between 50 to 60 years mostly having mobile phones as compare to older. He explained that they do not contact with the patients, all come to hospital and get there check up and get other appointment date from them. He said if patients come to their private clinics which is very common trend in our country so we have their mobile numbers, and we contact them if needed but mostly we do not.

\section{CONCLUSION}

The main objective of our study was to find out the functionalities of the mobile phone for elder persons who facilitate them and give them a happier, independent, secure, and joyful life. On the basis of informal discussions we designed the questionnaire for surveys and interviews. The surveys were conducted with the elder persons belongs to county of Blekinge for understanding the needs of elderly.

The two interviews conducted with the health care professionals. The findings from the questionnaire is statistically analyzed and obtained the concrete and accurate results. The results from interviews and questionnaires validated with the previous studies in same context. The research question was focused on those elderly who are facing diabetes type 2 . We have asked the reasons from them 
why they do not use mobile phones in their regular life. We came to know with the responses that some elder person do not like mobile phones where as some told that the complexity of mobile phone operating systems isolates them from mobile phones. The third and most important question of our study was about the functionality of mobile phone which elder people want in future mobile phones. The answers were quite similar about the functionality; most of them suggested that there should be less functional complexity in mobile phones. So, the elder people take interests in using mobile phone.

After conducting the surveys and planning the interviews. We found that the elder people avoid to use those devices which have complex functionalities and hard to understand. Some important points about the functionality of mobile phones which we have raised during surveys and interviews were appreciated by the respondents and they answered closer to our study. We have tried to pull out the accurate results and statistics to answer the research questions.

\section{REFERENCES}

[1] M. Conci, F. Pianesi, and M. Zancanaro, "Useful, social and enjoyable: Mobile phone adoption by older people," in Proceedings of the 12th
IFIP TC 13 International Conference on Human-Computer Interaction: Part I, pp. 63-76, 2009.

[2] Magnitude and Speed of Population Ageing, World Population Ageing 1950-2050. Population Division, DESA, United Nations.

[3] NIDDK [Online].

Available: http://diabetes.niddk.nih.gov/dm/pubs/overview/

[4] M. E. Sanchez, "Effects of questionnaire design on the quality of survey data," Public Opin Q, vol. 56, no. 2, pp. 206-217, 1992.

[5] C. B. Seaman, "Qualitative methods in empirical studies of software engineering," IEEE Transactions on Software Engineering, vol. 25, no. 4, pp. 557-572, Jul/Aug 1999.

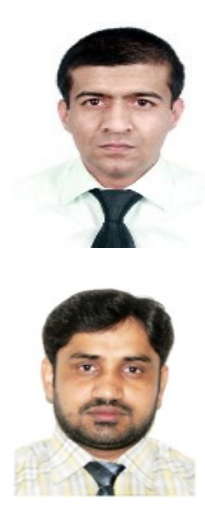

Farrukh Sahar was with Blekinge Institute of Technology, SE- 37179 Karlskrona, Sweden.

Syed Tosif Raza was with Blekinge Institute of Technology, SE- 37179 Karlskrona, Sweden. He is now with the World Health Organization, Regional Office for Europe Copenhagen. 